\title{
Time-Dependent Tracheal Deformation in Fetal, Neonatal, and Adult Rabbits
}

\author{
VINOD K. BHUTANI ${ }^{(17)}$ AND THOMAS H. SHAFFER \\ Department of Physiology, Temple University School of Medicine, Philadelphia, PA, USA
}

\begin{abstract}
Summary
Sequential magnitude and the rate of change in tracheal mechanics after application of intermittent positive pressure (IPP), and the time constant, $\tau$, of this deformation were determined in fetal (21 and 27 days gestation), neonatal (term, 31 days gestation) and adult rabbits. In vitro tracheal mechanics were determined by liquid plethysmography before and after IPP, $\left(25 \mathrm{~cm} \mathrm{H}_{2} \mathrm{O}\right.$ at 30 cycles/min) of $20,40,60$, and $120 \mathrm{~min}$ duration. A sequential decrease in tracheal compliance was documented at all ages. This was associated with increased values of $\tau$ as the maturational age advanced. Further, the rate of tracheal deformation was $0.008 \mathrm{~cm}$ $\mathrm{H}_{2} \mathrm{O} \mathrm{min}^{-1}$ at 21 days gestation compared to $0.00025 \mathrm{~cm} \mathrm{H}_{2} \mathrm{O}$ $\min ^{-1}$ at adulthood. The decrease in rate of deformation was related to the gestational maturity and duration of IPP. The rate of deformation, as predicted by calculated deformation time constants, appears to be a function of structural elastic and viscoelastic properties of the trachea as well as the duration and magnitude of applied pressure.
\end{abstract}

\section{Speculation}

The deformation time constant may be defined as a useful index to predict the rate of airway deformation. Utilizing the rabbit model, this provides a measure of comparing barotrauma with different modes of ventilation in preterms.

Pressure-induced deformation of the neonatal conducting airways have been attributed as a sequelae of intermittent positive pressure (IPP) application in growing neonates $(11,14)$ and in vitro experiments $(4,5)$. These experiments have suggested that the extent of barotrauma is best quantified by tracheal mechanics (4). Data on the sequentional mechanical properties of the airways would be pertinent to identify the magnitude and rate of tracheal deformation subsequent to IPP. Presently, these data are unavailable in the literature and this study was specifically designed to quantitate the magnitude and rate of tracheal deformation in fetal, neonatal, and adult rabbit tracheal segments. Based on these data and regression analysis, the deformation time constants were determined for specific gestational ages and the duration of IPP.

\section{MATERIALS-AND METHODS}

Experiments were performed to determine tracheal mechanics by liquid plethysmography. The preparation of isolated tracheal segments and the technique of plethysmography to determine tracheal pressure-volume relationships have been previously described (4). The study was designed in four groups defined by the postconceptual age of the New Zealand white rabbits and Group I and II were fetal tracheal segments at 21 and 27 days gestational age, respectively. Group III comprised trachea from term (31 \pm 1 days gestational age) neonates, and Group IV consisted of young adults at $18 \pm 6$ months age. The animals were anesthetized with sodium pentobarbital $(20 \mathrm{mg} / \mathrm{kg}$ by intravenous or intraperitoneal administration) and sacrificed with a bolus of magnesium chloride. Entire extrathoracic tracheal segments were dissected from a midline cervical incision.

IPP was applied to the tracheal segments while these were suspended in an isotonic saline bath as previously described (5). Zero to $25 \mathrm{~cm} \mathrm{H}_{2} \mathrm{O}$ inflating pressure was cycled at $30 /$ min utilizing a small animal Harvard ventilator. For each group, the duration of IPP was maintained for $20 \mathrm{~min}$ (four segments); $40 \mathrm{~min}$ (four segments); $60 \mathrm{~min}$ (six segments); and $120 \mathrm{~min}$ (six segments). Data were collected at these time intervals and compared to control data (six segments).

Mean internal tracheal diameters and lengths of the preparation were measured and used to calculate their respective volumes.

The resting length of the preparation at ambient atmospheric pressure was measured, with the aid of calipers, as a distance between the proximal and distal ligatures. Before ligation of the distal end of the segment, a fine tracheal section was obtained to measure its diameter. These sections were bathed in isotonic saline and minimally manipulated before the measurements were obtained. The diameter was determined directly in two different axes, and in addition the circumference was measured to recalculate the diameter indirectly (4). The changes in dimensions following IPP were calculated and expressed as a \% change over the control (nonventilated) dimensions.

Pressure-volume relationships of these segments were determined by liquid plethysmography at sequential periods of IPP (4). Pressure-volume changes were produced in stepwise pressure increments (or decrements) 5-10 $\mathrm{cmH}_{2} \mathrm{O}$. Inflation was commenced at atmospheric pressure and increased to a maximal $60-80 \mathrm{cmH}_{2} \mathrm{O}$. The segment was deflated in similar small decrements to a maximal -30 to $-50 \mathrm{cmH}_{2} \mathrm{O}$ or until no further volume change occurred. The preparation was then gradually reinflated to atmospheric pressure. Volume changes expressed as a \% of the resting volume and corresponding pressures were determined and plotted to provide individual quasi-static pressure-volume relationships. Specific tracheal complicance $\left(\mathrm{C}_{\mathrm{s}}\right)$ was determined as a function of the resting volume at that particular period of IPP. Tracheal $\mathrm{C}_{s}$ was measured as the relative change in resting volume over a pressure range of $0-10 \mathrm{cmH}_{2} \mathrm{O}$ on the deflation limb of the pressure-volume curves; this represents the linear portion of the deflation curve for all groups. (4).

Sequential deflation limbs of the pressure-volume loop were compared to quantitate the changes in $C_{s}$ as a function of IPP duration. Linear regression analysis of these data were obtained to predict the magnitude of tracheal deformation and time constant, $\tau$. These data were further differentiated to quantitate the rate of tracheal deformation for each group. Significant limits for the coefficient of regression $(r)$ were obtained when the population coefficient was zero with $(n-1)$ degrees of freedom, where $n=$ number of samples (10).

\section{RESULTS}

Sigmoid pressure-volume (P-V) curves were obtained with characteristic hysteresis during inflation and deflation of each tracheal 
segment $(3,4)$. Sequential deflation limbs following 20,40, 60, and $120 \mathrm{~min}$. IPP are shown in Figure 1 for preparations in Group II. These illustrate typical observations of sequential decrease in both the distensibility and slope of the curves with increasing duration of IPP. The magnitude of the changes were largest in Group I and regressed with advancing age groups.

Utilizing the P-V curves, $C_{s}$ was computed. The sequential mean data for all groups is shown in Figure 2. The exponential decrease in $\mathrm{C}_{s}$ was dramatic in Group I, whereas in Groups II, III, and IV there was a progressive decrease with the duration of IPP. In all groups the major proportion of the altered compliance occurred during the initial application of IPP, with a subsequent gradual decrease in $\mathrm{C}_{s}$ with increasing duration of IPP. Linear regression analysis of these data could be expressed as:

$$
\mathrm{C}_{\mathrm{s}}^{(\mathrm{t})}=\mathrm{C}_{\mathrm{s}} \mathrm{e}^{-\mathrm{t} / \tau}
$$

Equation 1

where, $C_{s}{ }^{(t)}$ and $C_{s}$ are the specific tracheal compliance at time ' $t$ ' min and 0 min respectively, and $\tau$ is the deformation time constant. Table 1 tabulates the equations and the coefficient of regression obtained for each of the four groups. The time constant of the tracheal segment increased with advancing age of the animal model and exhibited a 9-fold increase from 21 days gestation to adulthood (Fig. 3). A linear increase in $\tau$ was documented during fetal development of 21 days gestation to 31 days gestation.

Regression analysis in equation 1 was further differentiated:

$$
\frac{\mathrm{d}\left[\mathrm{C}_{\mathrm{s}}{ }^{(\mathrm{t})}\right]}{\mathrm{dt}}=\frac{-1}{\tau} \mathrm{C}_{\mathrm{s}} \mathrm{e}^{-\mathrm{t} / \tau}
$$

Equation 2

to define the rate of tracheal deformation. Theoretic curves of these equations were developed for all groups and are illustrated in Figure 4, such that the rate of tracheal deformation, determined by the term $\frac{\mathrm{d}\left[\mathrm{C}_{\mathrm{s}}{ }^{(t)}\right]}{\mathrm{dt}}$ (change in $\mathrm{C}_{\mathrm{s}}$ as a function of time: $\left[\mathrm{cmH}_{2} \mathrm{O}\right.$. $\mathrm{min}]^{-1}$ ) is correlated to the duration of IPP. In Group I, the rate was over $0.008 \mathrm{cmH}_{2} \mathrm{O}$ min at the onset on IPP and decreased to $0.0036 \mathrm{cmH}_{2} \mathrm{O} \mathrm{min}-1$ by $2 \mathrm{~h}$ of IPP. These values are higher than those for Group II, III, and IV. The adult group exhibited minimal deformation with a rate of $0.00025 \mathrm{cmH}_{2} \mathrm{O} \mathrm{min}^{-1}$ at the onset and

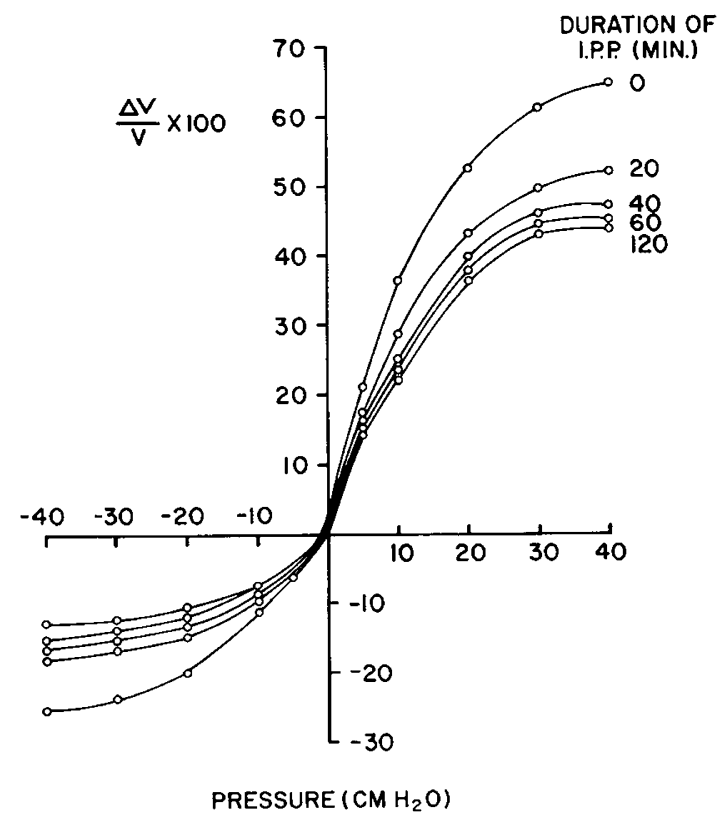

Fig. 1. Typical sequential deflation limbs pressure-volume curves of rabbit tracheal segments in Group II [27 days of intermittent partial pressure (IPP) gestation] after $0,20,40,60$, and $120 \mathrm{~min}$. The intraluminal pressure is on the abscissa. The relative change in volume, expressed as $\%$ of resting volumesa, is on the ordinate.

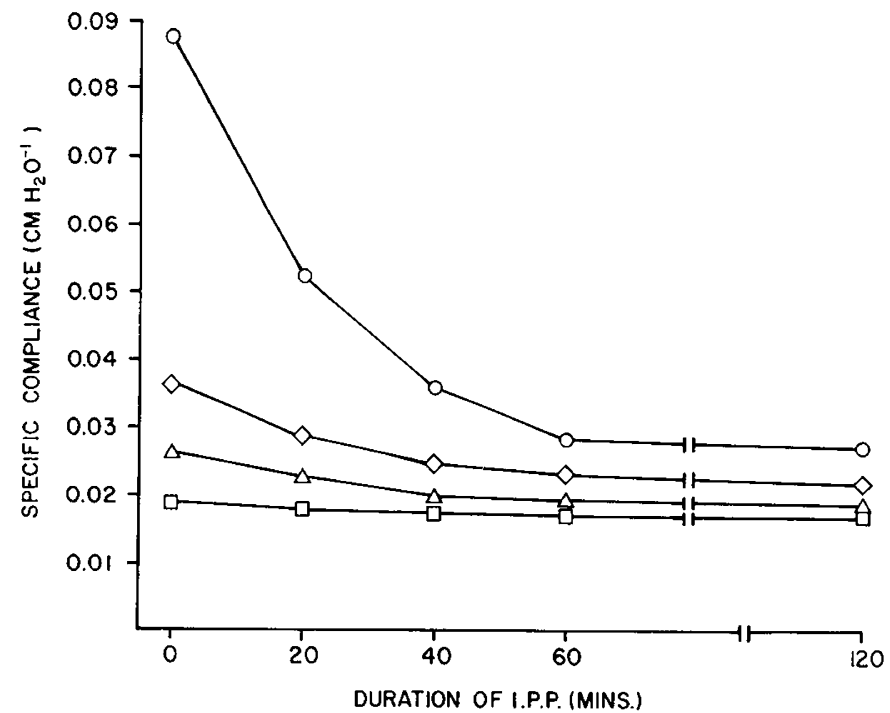

Fig. 2. Sequential changes in mean specific tracheal compliance at 0 $10 \mathrm{cmH}_{2} \mathrm{O}^{-1}$ as a function of duration of intermittent partial pressure (IPP). 0,21 day of gestation fetus; $\diamond, 27$ day of gestation fetus; $\Delta, 31$ day of gestation, term neonate; and $\square$, adult. (Lines represent best visual curves).

Table 1. Regression analyses of change in specific tracheal compliance after intermittent positive pressure

\begin{tabular}{clcc}
\hline Group & Equation & $\begin{array}{c}\text { Coefficient of } \\
\text { regression }\end{array}$ & $\begin{array}{c}\text { Limits of } \\
\text { significance }\end{array}$ \\
\hline I & $\mathrm{C}_{s}{ }^{(t)}=0.074 \mathrm{e}^{-t / 85.9}$ & $r=0.90$ & $P<0.001$ \\
II & $\mathrm{C}_{s}{ }^{(\mathrm{t})}=0.032 \mathrm{e}^{-\mathrm{t} / 237.2}$ & $r=0.88$ & $P<0.001$ \\
III & $\mathrm{C}_{\mathrm{s}}{ }^{(\mathrm{t})}=0.029 \mathrm{e}^{-\mathrm{t} / 296.0}$ & $r=0.96$ & $P<0.001$ \\
IV & $\mathrm{C}_{\mathrm{s}}{ }^{(\mathrm{t})}=0.017 \mathrm{e}^{-\mathrm{t} / 756.6}$ & $r=0.95$ & $P<0.001$ \\
\hline
\end{tabular}

${ }^{1}$ See text for explanation.

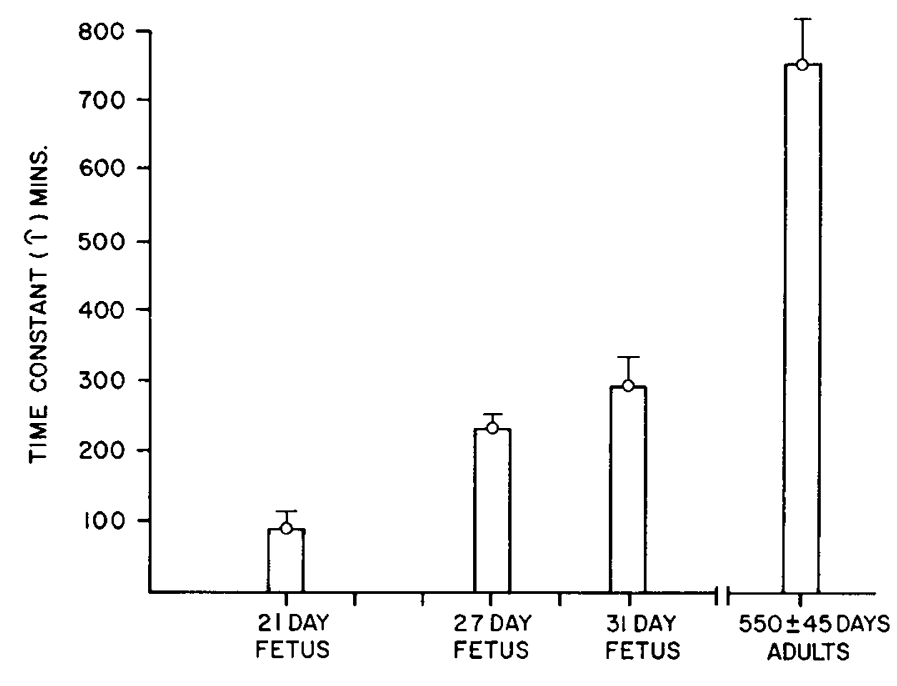

DEVELOPMENTAL AGE

Fig. 3. Time constant $(\tau)$ (mean \pm S.E.) of tracheal deformation as a function of developmental age of rabbit.

almost a similar rate atter $2 \mathrm{~h}$ of IPP. Rate of deformation was highest at the beginning of IPP in all groups and the magnitude of decrease was related to the maturity of the model and duration of IPP.

Concomitant to the on going changes in $\mathrm{C}_{\mathrm{s}}$, the tracheal dimensions exhibited an increase after IPP. The sequential \% increase over control values in tracheal diameter, length, and volume after 
$20,40,60$, and $120 \mathrm{~min}$ of IPP are tabulated for all groups in Table 2 .

\section{DISCUSSION}

The present study demonstrates that sequential mechanical and dimensional deformation of the fetal and neonatal trachea occur with the application of IPP. Utilizing untethered excised tracheal segments we have shown the sequential decline in tracheal compliance. Most of the change was observed in the first $20-40 \mathrm{~min}$ of IPP and followed by a steady, progressive but gradual decrease. This has been observed to be a predictable trend in all four groups, and the magnitude of change decreases with advancing age. These data are in agreement with our previous observations of significant pressure-induced deformation being sustained by more susceptible

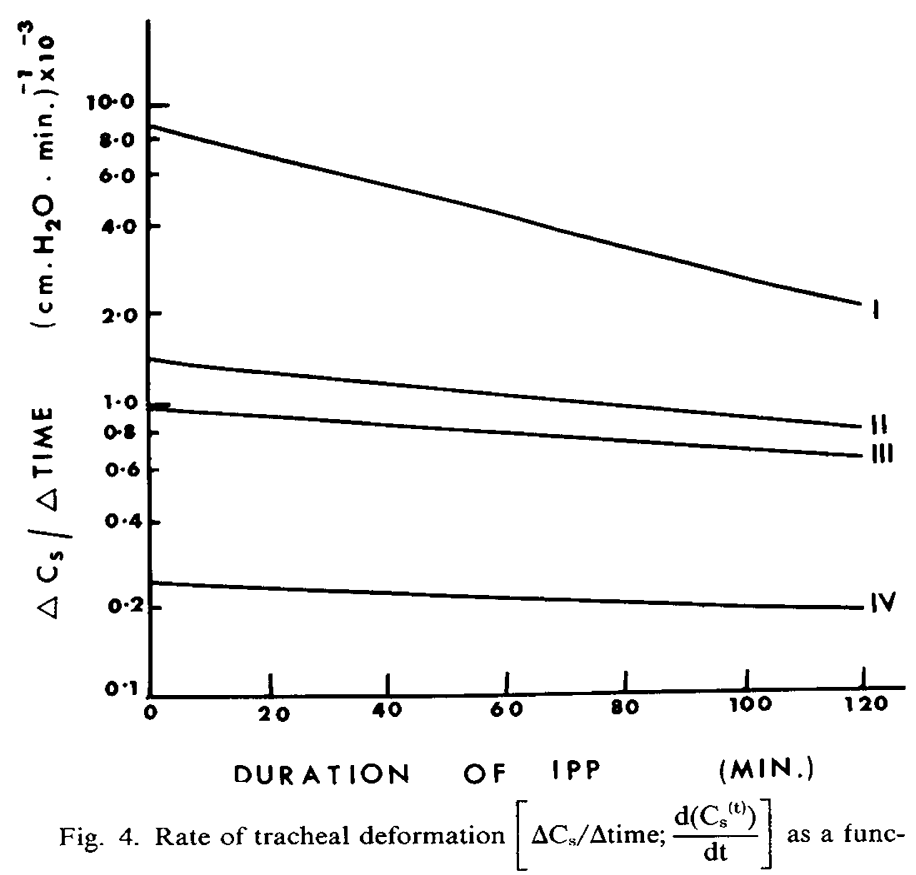

tion of duration of intermittent partial pressure (IPP). Theoretical relationships calculated for Group I, II, III, and IV.

Table 2. Sequential mean \% increase in diameter, length and volume of tracheal segments after intermittent positive pressure ${ }^{1}$

\begin{tabular}{crccc}
\hline & \multicolumn{4}{c}{ Duration (min) } \\
\cline { 2 - 5 } & \multicolumn{1}{c}{20} & \multicolumn{2}{c}{40} & \multicolumn{1}{c}{60} \\
\hline Diameter & & & 120 \\
Groups & & & \\
I & $18.7 \pm 2.4$ & $30.6 \pm 3.2$ & $40.0 \pm 3.6$ & $46.7 \pm 4.2$ \\
II & $3.4 \pm 0.4$ & $16.5 \pm 2.1$ & $23.0 \pm 2.5$ & $22.0 \pm 2.4$ \\
III & $8.3 \pm 0.9$ & $15.2 \pm 1.2$ & $19.1 \pm 0.8$ & $22.0 \pm 2.2$ \\
IV & $0.7 \pm 0.2$ & $2.3 \pm 0.2$ & $3.2 \pm 0.2$ & $3.7 \pm 0.2$ \\
Length & & & & \\
Groups & & & & \\
I & $6.3 \pm 0.6$ & $11.7 \pm 0.9$ & $18.2 \pm 1.9$ & $19.0 \pm 2.4$ \\
II & $5.6 \pm 0.6$ & $14.6 \pm 1.6$ & $16.5 \pm 1.0$ & $19.5 \pm 2.0$ \\
III & $2.6 \pm 0.2$ & $5.4 \pm 0.3$ & $9.8 \pm 0.8$ & $9.9 \pm 0.8$ \\
IV & $2.5 \pm 0.2$ & $4.5 \pm 0.2$ & $4.2 \pm 0.3$ & $6.7 \pm 0.5$ \\
Volume & & & & \\
Groups & & & & \\
I & $38.9 \pm 4.1$ & $49.0 \pm 5.0$ & $124.0 \pm 12.1$ & $132.9 \pm 14.2$ \\
II & $8.0 \pm 0.8$ & $49.0 \pm 4.8$ & $74.0 \pm 6.3$ & $86.0 \pm 9.1$ \\
III & $20.3 \pm 2.1$ & $39.3 \pm 3.6$ & $56.0 \pm 4.9$ & $65.0 \pm 7.3$ \\
IV & $2.9 \pm 0.2$ & $8.5 \pm 0.9$ & $12.0 \pm 1.2$ & $14.0 \pm 1.2$ \\
\hline
\end{tabular}

\footnotetext{
${ }^{1}$ Data are \% increase over control values of diameter, length volume at time $=0 \mathrm{~min}$ of intermittent positive pressure.
}

immature airways after short duration of IPP and continuous positive airway pressure (CPAP) $(4,5)$. The deformation time constant, calculated from these data, represents the ability of the trachea to withstand the stress and strain of IPP. As shown, this capability increases with advancing maturational age; a 3-fold increase occurs between 21-27 days postconceptional age and continues to increase postnatally. In addition, the dimensional deformation corroborate the significant changes previously reported (4).

Anatomic maturation of the airways is associated with decreasing tracheal compliance and changing mechanical properties of the fetal and neonatal airways $(3,8)$. This is manifested by increasing amounts of smooth muscle, collagen, and cartilaginous tissue components (12) and is similar to the increase in collagen and collagen-to-elastin ratio of arterial walls during development and aging (11). The resultant stiffening provides the structural support and resistance to distension and collapse during intraluminal pressure oscillation during a respiratory cycle. The circumferential and longitudinal distension are a result of the stress encountered by the airway walls, in particular by the pars membrana. The ability of a structure to withstand stress has been documented by its elastic modulus. Of these, the bulk modulus of the trachea has been shown to increase with advancing gestational age (15). Thus, unlike the adult trachea, the highly compliant fetal or neonatal trachea would be more prone to the stress of prolonged IPP.

During repeated static P-V determinations the airway wall compliance has been observed to decrease $(1,13)$. This phenomenon has been attributed to previous stretching, as well as the frequency and volume history after repeated elastic loading of the airways (13). Repetitive stretching as in a constant physiologic pressure oscillation has been observed in the aorta as it dilates and functionally stiffens with advancing age (2). Similar increased stiffness and distension has been observed in both in vitro and in vivo arterial preparations (11). Changes in the mechanical properties of vessels to low frequency pressure oscillation have been attributed to the extensibility of retracted elastin at small extensions, and the stiffness of collagen at larger extensions (7). The load bearing properties of the individual tissue components differ from each other and account for their variable resistance to distension and deformation. The change in the mechanical properties of the airway wall to the stimulus of pressure oscillation would be comparable to structural fatigue of metal. The rate of such deformation can be described by theoretic relationships and has been established for fetal and neonatal rabbit tracheal segments in the present study.

Bobbaers et al. (6) have documented that the viscous resistance of the adult canine trachea is about $1 \%$ of its dynamic elastance. The resistance has been suggested to be related to the amount of tracheal smooth muscle (9). Because the amount of trachealis muscle has been known to be related to developmental maturation, the magnitude of tracheal deformation would be at earlier gestational ages. Similarly, at these ages, because of the high static tracheal compliance (low elastance), one would assume a greater susceptibility to deformation and an associated increased rate of deformation. Finally, it has been shown that both the dynamic elastance and viscous resistance increase with stretching (6); thus, as tracheal dimensions increase during stretching (during IPP), because of the increased elastance and resistance, the rate of deformation would decrease with time. This has been demonstrated in Figure 4. The role of fetal tracheal elastance and resistance needs to be correlated to the structural components of the tracheal and investigated further.

In conclusion, by correlating the magnitude and duration of applied pressure to the magnitude and rate of tracheal deformation, to the time constants, and to the mechanical properties of the trachea one may compare and assess barotrauma induced by different modalities of ventilatory support.

\section{REFERENCES AND NOTES}

1. Akhtar, A. J., Merchant, S., McHardy, G. J. R. and Crofton, J. W.: Measurement of bronchial elastance in resected sheep lungs. Thorax, 28: 107 (1973). 
2. Bader, $\mathbf{H}$.: Dependence of wall stress in the human thoracic aorta on age and pressure. Circ. Res., 20: 354 (1967).

3. Bhutani, V. K., Rubenstein, S. D., and Shaffer, T. H.: Pressure-volume relationships of trachea in fetal, neonatal and adult rabbits. Resp. Physiol., 43: 221 (1981).

4. Bhutani, V. K., Rubenstein, S. D., and Shaffer, T. H.: Pressure-induced deformation in immature airways. Pediatr. Res., 15: 829 (1981).

5. Bhutani, V. K. and Shaffer, T. H.: Effect of intermittent positive pressure application on the bulk modulus of the developing rabbit trachea. Respiration, 4I(3): 192 (1981).

6. Bobbaers, H., Clement, H. J.,and van de Woestijne, K. P.: Dynamic viscoelastic properties of the canine trachea. J. Appl. Physiol., Respirat. Environ. Exercise Physiol., 44(2): 137 (1978).

7. Boughner, D. R. and Roach, M. R.: Effect of low frequency vibration on the arterial wall. Circ. Res., 29: 136 (1971)

8. Burnard, E. d., Grattan-Smith, P., Picton-Warlow, C. G., and Grauaug, A.: Pulmonary insufficiency in prematurity. Aust. Pediatr. J., l: 12 (1965).

9. Coburn, R. F. D., Thornton, D., and Arts, R.: Effect of trachealis muscle contraction on tracheal resistance to airflow. J. Appl. Physiol., 32: 397 (1972).
10. Diem, K. (Ed.): Scientific Tables; Geigy Chemical Corporation. p. 61 (1962).

11. Dobrin, P. R.: Mechanical properties of arteries. Physiol. Rev., 58(2): 397 (1978)

12. Engle, S.: The Prenatal Lung, Glasgow, p. 37 (Pergamon Press, 1966).

13. Martin, H. B. and Proctor, D. R.: Pressure-volume measurements on dog bronchi J. Appl. Physiol., 13: 337 (1975).

14. Northway, W. H., Rosan, R. C., and Porter, D. Y.: Pulmonary disease following respirator therapy of hyaline membrane disease: bronchopulmonary dysplasia. N. Engl. J. Med., 276: 357 (1967)

15. Shaffer, T. H. and Bhutani, V. K.: Alterations in bulk modulus of trachea during development. Respiration, 39: 344 (1980).

16. The authors are grateful for the secretarial assistance of Mary O'Connor. The research leading to the article was supported in part by Public Health Service Grant \#HL-22843.

17. Requests for reprints should be addressed to: Dr. Vinod K. Bhutani, Department of Physiology, Medical Research Bldg. Room 224, 3240 N. Broad Street, Philadelphia, PA 19140.

18. Received for publication November $5,1981$.

19. Accepted for publication April 16, 1982. 